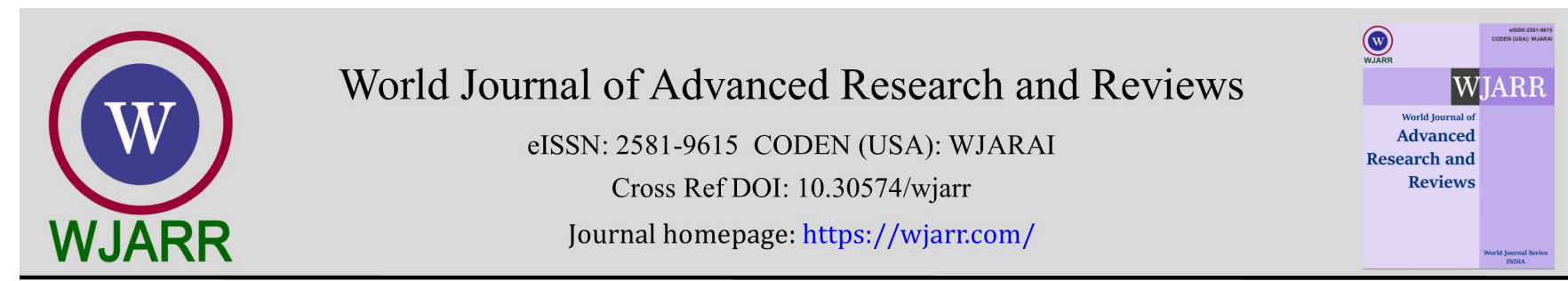

(REVIEW ARTICLE)

Check for updates

\title{
The evolving nature of asthma and contemporary management of this respiratory disorder
}

\author{
Andrew Kiboneka * \\ Department of Paediatrics, Case Hospital, Uganda.
}

World Journal of Advanced Research and Reviews, 2021, 10(01), 162-167

Publication history: Received on 09 March 2021; revised on 12 April 2021; accepted on 15 April 2021

Article DOI: https://doi.org/10.30574/wjarr.2021.10.1.0154

\begin{abstract}
Asthma is a complex common heterogenous syndrome that often starts in childhood and is chacterized by cough wheezing and increased airway responsiveness to a variety of environmental stimuli. The definition of asthma involves various phenotypes and endotypes.The immunopathogenesis of asthma involves many immune cell sand involves the airway epithelium as well as both innate and adaptive components of the immune system. Understanding the Immunology of asthma is essential in its treatment.Asthma is a rising significant global public health burden. The annual prevalence of severe asthma episodes is estimated from $1 \%$ to $21 \%$ for adults and over $20 \%$ for children aged 6-7 years. The prevalence of asthma varies widely around the world, ranging from $0.2 \%$ to $21.0 \%$ in adults and from $2.8 \%$ to $37.6 \%$ in 6- to 7-year-old children. The development of novel asthma phenotyping \& endo typing plus better classification of patients using machine learning and big data have markedly improved asthma treatment outcomes in both children and Adults.Several research groups have developed cluster analyses of phenotypes and endo-types in severe asthma. These clusters support the importance of disease heterogeneity in asthma and suggest differences in pathophysiologic mechanisms that define these clusters.Biomarkers of asthma include Serum Immunoglobulin, Fractional excretion of nitric oxide and blood eosinophils amongst others. There are five food and drug administration approved biologicals for the treatment of severe asthma.
\end{abstract}

Keywords: Asthma; Th2 High; Th2low; Phenotypes; Endotypes, Clusters, Biomarkers, Biologicals; Bronchial Thermoplasty.

\section{Introduction}

Asthma is a complex common heterogenous syndrome that often starts in childhood and is chacterized by cough wheezing and increased airway responsiveness to a variety of environmental stimuli. The definition of asthma involves various phenotypes and endotypes. [1,2]. Asthma is defined as a clinical syndrome of intermittent respiratory symptoms triggered by viral upper respiratory infections, environmental allergens, or other stimuli, and is characterized by nonspecific bronchial hyperresponsiveness and airways inflammation [3] Airway epithelial cells (ECs) lie at the interface between the host and the environment and represent the first line of defense against microorganisms, gases and allergens [4].. In their exposed position, ECs express many pattern recognition receptors [PRRs] to rapidly detect and respond to pathogen-associated molecular patterns (PAMP)] found in microbes or to damage-associated molecular patterns (DAMP)] released upon tissue damage, cell death or cellular stress. The activation of epithelial PRRs leads to the release of cytokines, chemokines and antimicrobial peptides that attract and activate innate and adaptive immune cells. Recent studies have demonstrated that EC activation is a key triggering event in the recognition of inhaled allergens that activates the local network of dendritic cells (DCs), which coordinate the subsequent immune response [5].

\footnotetext{
${ }^{*}$ Corresponding author: Dr Andrew Kiboneka

Department of Paediatrics, Case Hospital, Uganda.
}

Copyright (C) 2021 Author(s) retain the copyright of this article. This article is published under the terms of the Creative Commons Attribution Liscense 4.0. 


\subsection{Definition of severe asthma for patients $>6$ years of age}

Asthma which requires treatment with guidelines suggested medications for GINA steps 4-5 asthma (high dose ICS\# and LABA or leukotriene modifier/theophylline) for the previous year or systemic CS for $050 \%$ of the previous year to prevent it from becoming "uncontrolled" or which remains "uncontrolled" despite this therapy [1]

\section{The evolving nature of asthma, phenotypes, endotypes and clusters of severe asthma}

The past 30 years have seen major advances in the understanding of asthma mechanisms and the clinical introduction of effective medicines based on these pathways. The first major step-change occurred in the 1980s with the development of bronchial challenge as a research tool to dissect the airways inflammatory responses [6,7]. The advent of bronchoscopy and sputum induction in the early 1990s, enabled the description of characteristic features of airways inflammation and remodeling [8-11)], while epidemiological and challenge studies showed viruses to be the main cause of asthma exacerbations One of the greatest discoveries was the central role of Type-2 cytokines driven by the interleukin [IL]-4/IL-13 and IL-5 pathways, for which simple biomarkers, like sputum eosinophils and exhaled nitric oxide, were discovered. However, it was not until early 2000s that the basic science translated into fundamentally novel treatments, beginning the "era of biological treatments" with the anti-Immunoglobulin E antibody, omalizumab. It took almost 15 years for the next biologics to arrive, targeting the pro-eosinophilic cytokine IL-5, showing how difficult drug development can be.

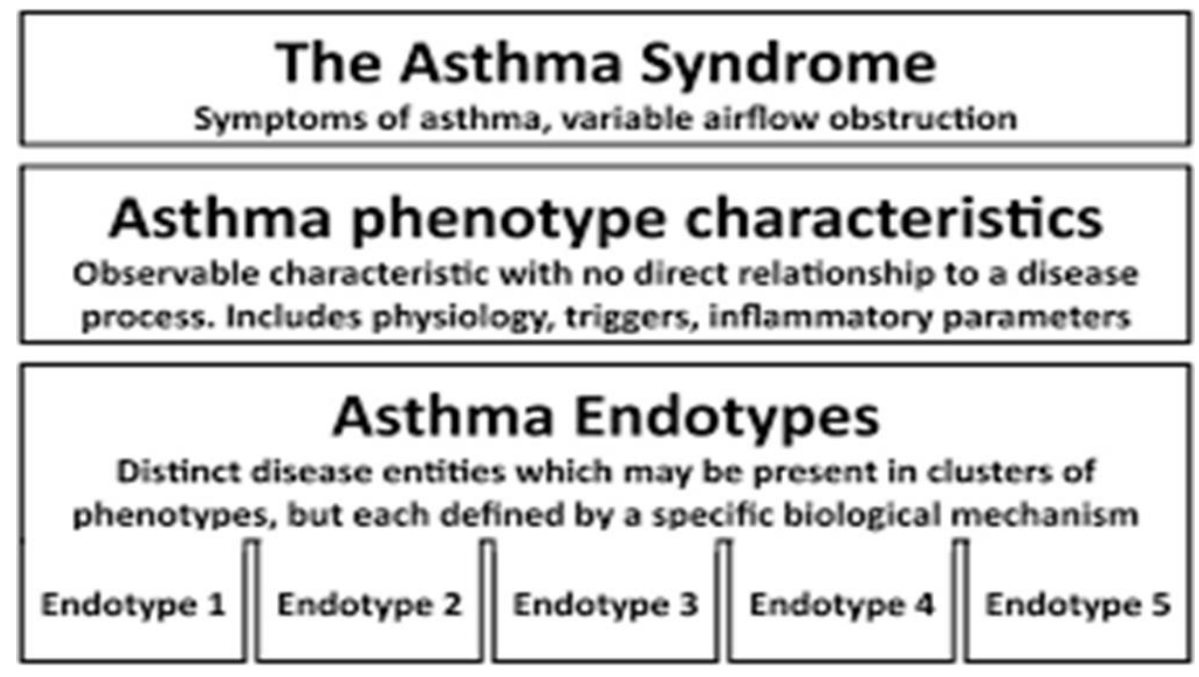

Figure 1 Asthma phenotypes \& endotypes.

We now find ourselves in a fortunate, yet challenging, situation: we have several biologics to offer to people with asthma, yet we do not have clinical algorithms to identify the "right drug for the right patient at the right time". Moreover, these biologics are constrained to Type-2 high asthma and we lack effective treatments for other phenotypes, such as neutrophil-variant and pauci-granulocytic asthma. These issues of key importance to people with asthma, their clinicians, the laboratory scientists, the pharmaceutical industry, regulators and payers, gain even more importance with the recognition that severe asthma is heterogeneous both in clinical and pathological terms In Europe, the effort to stratify asthma was led by the Innovative Medicines Initiative (IMI)-funded consortium U-BIOPRED (Unbiased BIOmarkers for the Prediction of REspiratory Disease outcomes) resulting in major discoveries that, together with those from the US consortium, SARP (Severe Asthma Research Program), have transformed our understanding of asthma mechanisms and provided new clinical asthma phenotypes They open up the possibility of discovering the underlying driving molecular endotypes behind these variants. It is clear, however, that only large integrated data strategies will succeed in realizing this ambition [.12] 


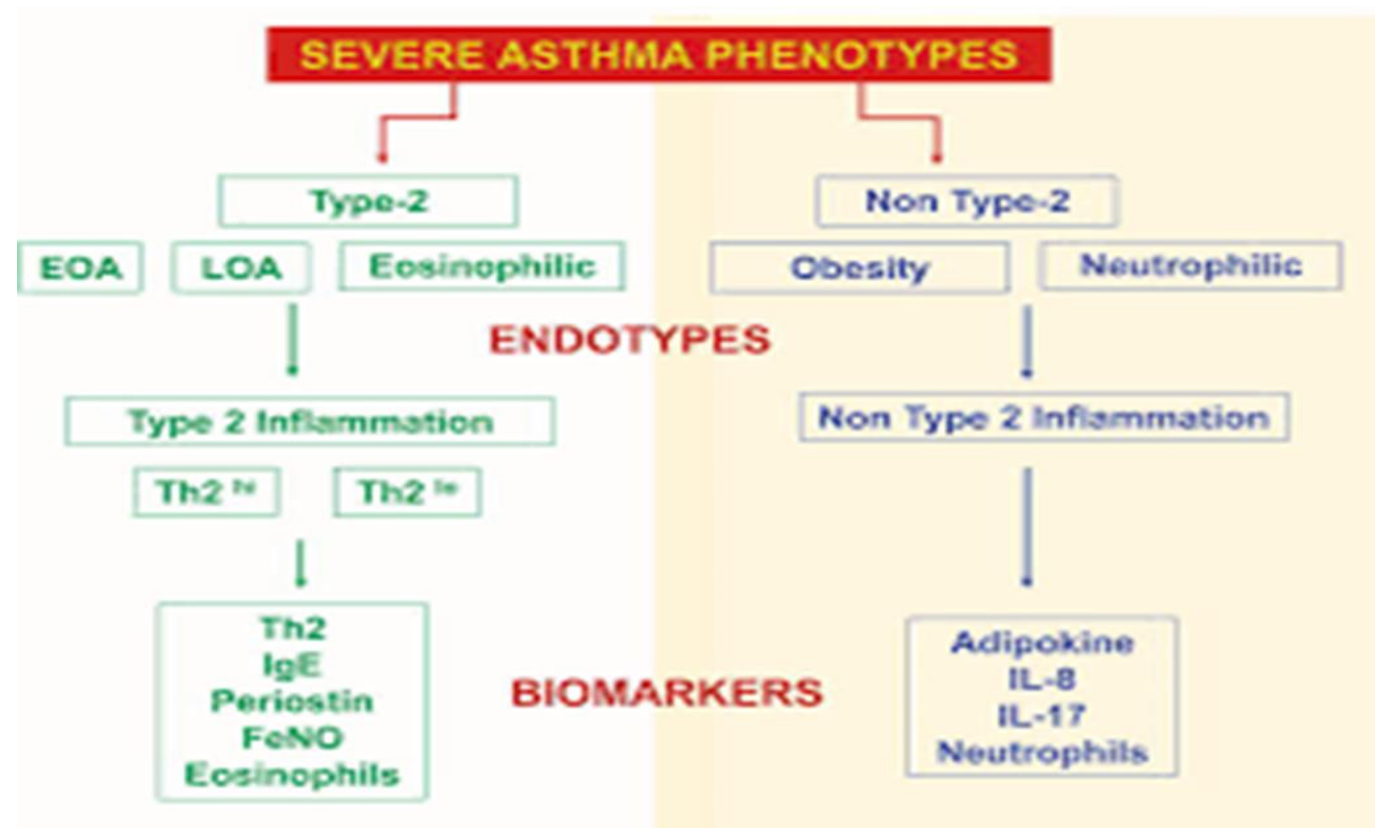

Figure 2 Classification of severe asthma

\section{The immunology of asthma}

More recent data indicate that the categorization into allergic Immunoglobulin E-mediated and non-allergic nonImmunoglobulin E-mediated phenotypes is oversimplifying the complex situation in asthmatic patients. Although the majority of asthma patients are well controlled with anti-inflammatory medications including inhaled corticosteroids (ICS) or leukotriene receptor antagonists together with short and/or long acting beta-adrenergic drugs, there is a substantial need for additional and new therapeutic approaches. Several studies indicate that about a third to a quarter of patients do not respond adequately to ICS, or are steroid resistant or develop side effects to conventional antiinflammatory therapy, thus limiting their use $[13,14]$. This situation has stimulated a series of investigations aiming to better understand the complex pathophysiology of the disease in order to target medication in a more specific way. This approach requires the identification of distinct signaling pathways and, subsequently, biomarkers, to test new drug developments which target and interfere in a highly specific and selective fashion with these cellular and molecular phenotypes.

The chronic inflammatory reaction is based on a complex interaction of different cell types, all of them belonging to the adaptive or innate immune system and including T-lymphocytes, mast cells, eosinophils and antigen-presenting cells and structural cells such as macrophages, dendritic cells, bronchial epithelium and myofibroblasts and their products.

As central cells in the regulation of allergic inflammation, eosinophils and Tymphocytes have the ability to differentiate from naïve TH0 cells to effector T cells under special conditions. The subsets of T-helper type 1 (TH1) and T-helper type 2 (TH2) cells are prototypical. An enhanced production of proinflammatory cytokines such as the interleukins IL-4, IL5 or IL-13 by the TH2 subset mediates pulmonary allergic inflammation. These T cells control the effector response at the level of the innate immune system (granulocytes, mast cells). Furthermore, they control the level of antibody production by the B-lymphocytes - in this case especially the Immunoglobulin E isotype. The TH2 subset plays a central role in regulation of the allergen-driven phenotype of $B A[15,16]$.

\subsection{The TH1-TH2 concept: differentiation of $\mathrm{T}$ lymphocytes}

Activation of epithelial cells leads to the secretion of cytokines IL-33 and IL-25, which subsequently promotes the release of IL13 and IL-5 from the IL-25-receptor-positive natural helper cells, which themselves induce the differentiation of naïve T cells into TH2 cells.

Activation of epithelial cells leads to the secretion of cytokines IL-33 and IL-25, which subsequently promotes the release of IL13 and IL-5 from the IL-25-receptor-positive natural helper cells, which themselves induce the differentiation of naïve T cells into TH2 cells. 
The induction of TH2 cells is prototypical for the development of atopic disorders; the preferential differentiation into the TH2 endotype is mediated through the interaction of genetic predisposition and environmental influences such as the microbiological milieu [15].

For the development of TH2 effector cells, the transcription factors STAT6 and GATA binding protein 3 (GATA3), which is induced in the presence of IL-4 and IL-6, play a critical role. GATA3 itself activates genes which encode for IL-3, IL-4, IL-5, IL-9, IL-13 and GM-CSF (15). GATA3 inhibits STAT-4 as well T-bet (the transcription factor which regulates the TH1-endotype), which results in TH2 polarization. Reversely, T-bet can inhibit GATA3 expression (15). The differentiation of T cells is essentially modulated by the prevalent milieu of cytokines. At the other end of the spectrum, the differentiation of TH0 cells into TH1 cells takes place.

Therefore IL-4 and IL-13 play a prominent role in Immunoglobulin E isotype switching in plasma cells. The allergenspecific Immunoglobulin E antibodies bind to high-affinity receptors expressed on basophils and mast cells, which degranulate after a second allergen contact following crosslinking of membrane-bound antibodies. This leads to a release of mediators of inflammation such as histamine, prostaglandins and leukotrienes from preformed granules as well to the de novo synthesis of soluble mediators of inflammation.

This represents the allergic early-phase reaction, which develops within just a few minutes of allergen contact and which presents clinically as an acute bronchial constriction that returns back to baseline levels within the next few hours. In the late-phase response, newly synthesized lipid mediators such as prostaglandins and leukotrienes appear together with an influx of eosinophils and T cells.

Also, a further aspect in a TH2-dominated effector response is the development of goblet-cell metaplasia, increased production of mucus and the development of fibrosis in the airways. Further consequences include hyperresponsiveness of the smooth muscle fibres. All of these are part of so-called airway remodelling.

Eosinophils play an essential role in the TH2-inflammatory response. In contrast to the early-phase allergic reaction, the asthmatic late-phase response is characterized by the dominance of this cell type. IL- 5 causes the sustained development and activation of eosinophil granulocytes in the bone marrow. After accumulation in the bronchial tissue, eosinophils release a broad spectrum of inflammatory mediators. The inflammation is maintained by different lipid mediators, cytokines, and other highly toxic mediators such as oxygen-free radicals, major basic protein (MBP), eosinophil-derived neurotoxin (EDN), platelet-activating factor (PAF), prostaglandins and especially the highly toxic eosinophilic cationic protein (ECP). Following chronic activation these mechanisms form the basis for airway remodelling, which is characterized by the destruction of the bronchial epithelium and hyperplasia of the smooth myocytes and glandular cells, as well as an enlargement of the bronchial basement membrane.

\subsection{TH2-high and TH2-low Asthma \& gene signatures and endotypes.}

Based on the above findings suggesting that the products of the POSTN, CLCA1 and SERPINB2 genes could serve as a surrogate marker for a TH2-driven immune-response, Woodruff et al. $[17,18]$ examined whether this gene signature can contribute to theidentification of different endotypes within the whole population of BA patients. The experiments and studies revealed that these three genes were over-expressed only in a subset of asthmatics. To define this subset precisely, unsupervised hierarchical clustering was applied to the findings of the gene array analysis based on the expression level of the genes POSTN, CLCA1 and SERPINB2.

Almost half of the subjects showed high expression level TH2-induced genes, while the other half exhibited a normal expression level. A comparison of all BA subjects and healthy controls revealed a general over-expression of the three genes. However, after hierarchical clustering about half of the asthma-patients remained undistinguished from the healthy controls using these surrogate markers. As a result, the following two clusters could be separated: in cluster 1 the markers POSTN, CLCA1 and SERPINB2were highly up-regulated, which was not the case in cluster 2, representing the other half of the participants and the healthy controls. These studies together with additional analysis of the characteristics of airway inflammation helped to define the TH2-high and the TH2-low endotypes. At one end of the spectrum it is possible to define an endotype with an up-regulated TH2 response mediated by the typical TH2 cytokines IL-5, IL-13 and IL-4 measured by the surrogate-markers POSTN, CLCA1 and SERPINB2; and at the other end resides the endotype which is not associated with a TH2 response.

Molecular endotyping obviously has important therapeutic implications. While the TH2 endotype, in particular the eosinophilic subtype, largely contains responders to steroid-treatment, TH2-low endotype asthma, which probably is consistent with the neutrophilic phenotype, is less understood with regard to the molecular pathogenesis. 


\section{Conclusion}

With the evolution of novel biological therapies, patients, who do not-respond to conventional asthma therapy require novel biologic medications, such as anti-Immunoglobulin E, anti-IL-5 and anti-IL4/IL13 to control asthma symptoms. Currently there are five food and drug administration approved biologics for asthma - omalizumab, mepolizumab, reslizumab, benralizumab, and dupilumab - with several others currently in development.

Omalizumab targets allergy antibodies known as Immunoglobulin E. Mepolizumab, reslizumab, and benralizumab all target pathways that affect eosinophils, a cell involved in allergic inflammation. Dupilumab targets a receptor for two molecules that drive allergic inflammation.

Asthma is associated with immune system activation, airway hyperresponsiveness (AHR), epithelial cell activation, mucus overproduction and airway remodeling. Both innate and adaptive immunity play roles in immunologic mechanisms of asthma. Type 2 asthma with eosinophilia is a common phenotype in asthma. It occurs with and without visible allergy. The type 2 endotype comprises; T helper type 2 (Th2) cells, type 2 innate lymphoid cells (ILC2), Immunoglobulin E-secreting B cells and eosinophils. Eosinophilic nonallergic asthma is ILC2 predominated, which produces IL-5 to recruit eosinophil into the mucosal airway. The second major subgroup of asthma is non-type 2 asthma, which contains heterogeneous group of endoypes and phenotypes, such as exercise-induced asthma, obesity induced asthma, etc. Neutrophilic asthma is not induced by allergens but can be induced by infections, cigarette smoke and pollution. IL-17 which is produced by Th17 cells and type 3 ILCs, can stimulate neutrophilic airway inflammation. Macrophages, dendritic cells and NKT cells are all capable of producing cytokines that are known to contribute in allergic and nonallergic asthma. Bronchial epithelial cell activation and release of cytokines, such as IL-33, IL-25 and TSLP play a major role in asthma. Especially, allergens or environmental exposure to toxic agents, such as pollutants, diesel exhaust, detergents may affect the epithelial barrier leading to asthma development. Biomarkers and Biologicals should be made more readily available in resource limited settings, which also experience a high burden of Asthma as elsewhere.

\section{Compliance with ethical standards}

\section{Acknowledgments}

Professor Peter Nyarango of the University of Namibia and Professor Russel Hopp of Creighton University, Omaha, Nebraska, U.S.A for their mentorship.

\section{Disclosure of conflict of interest}

The author confirms that this contents have no conflicts of interest.

\section{References}

[1] The Global Initiative for Asthma (GINA). Update. 2020.

[2] National Asthma Education and Prevention Program NAEPP. 2020.

[3] Busse WW, Lemanske RF Jr. Asthma. N Engl J Med. 2001;344:350-362.

[4] Xiao C, et al. Defective epithelial barrier function in asthma. J. Allergy Clin. Immunol. 2011; 128: 549-556.e1-12.

[5] Lambrecht BN, Hammad H. Biology of lung dendritic cells at the origin of asthma. Immunity. 2009; 31: 412-424.

[6] Cockcroft DW, Ruftin RE, Dolovich J, et al. Allergen-induced increase in non-allergic bronchial reactivity. Clin Allergy. 1977; 7: 503-513.

[7] De Monchy JGR, Kauffman HF, Venge P, et al. Bronchoalveolar eosinophilia during allergen-induced late asthmatic reaction. Am Rev Respir Dis. 1985; 131: 373-376.

[8] Djukanović R, Roche WR, Wilson JW, et al. Mucosal inflammation in asthma. Am Rev Respir Dis. 1990; 142:434457.

[9] Pin I, Gibson PG, Kolendowicz R, et al. Use of induced sputum cell counts to investigate airway inflammation in asthma. Thorax.1992; 47: 25-29. 
[10] Wardlaw AJ, Dunnette S, Gleich GJ, et al. Eosinophils and mast cells in bronchoalveolar lavage in subjects with mild asthma. Am Rev Respir Dis. 1988; 137: 62-69.

[11] Vignola AM, Kips J, Bousquet J. Tissue remodeling as a feature of persistent asthma. J Allergy Clin Immunol. 2000;105: 1041-1053.

[12] Djukanovic R, Adcock IM, Anderson G, et al. The Severe Heterogeneous Asthma Research collaboration, Patientcentred (SHARP) ERS Clinical Research Collaboration: a new dawn in asthma research. Eur Respir J. 2018; 52: 1801671.

[13] Szefler SJ, Martin RJ, King TS, Boushey HA, Cherniack RM, Chinchilli VM, et al. Significant variability in response to inhaled corticosteroids for persistent asthma. J Allergy Clin Immunol. 2002; 109(3):410-418.

[14] Martin RJ, Szefler SJ, King TS, Kraft M, Boushey HA, Chinchilli VM, et al. The Predicting Response to Inhaled Corticosteroid Efficacy (PRICE) trial. J Allergy Clin Immunol. 2007; 119(1):73-80.

[15] Holgate ST, Polosa R. Treatment strategies for allergy and asthma. Nat Rev Immunol. 2008; 8[3]:218-230.

[16] Bhakta NR, Woodruff PG. Human asthma phenotypes: from the clinic, to cytokines, and back again. Immunol Rev. 2011; 242(1):220-232.

[17] Woodruff PG, Boushey HA, Dolganov GM, Barker CS, Yang YH, Donnelly S, et al. Genome-wide profiling identifies epithelial cell genes associated with asthma and with treatment response to corticosteroids. Proc Natl Acad Sci USA. 2007; 104(40):15858-15863.

[18] Woodruff PG, Modrek B, Choy DF, Lia G, Abbas AR, Ellwanger A et al. T-helper type 2-driven inflammation defines major subphenotypes of asthma. Am J Respir Crit Care Med. 2009; 180(5):388-395. 